\title{
On the last factor of the period polynomial for finite fields
}

\author{
by
}

\section{S. Gurak (San Diego, Cal.)}

1. Introduction. Let $q=p^{a}$ be a power of a prime, and $e$ and $f$ positive integers such that ef $+1=q$. Let $\mathbb{F}_{q}$ denote the field of $q$ elements, $\mathbb{F}_{q}^{*}$ its multiplicative group and $g$ a fixed generator of $\mathbb{F}_{q}^{*}$. Let $\operatorname{Tr}: \mathbb{F}_{q} \rightarrow \mathbb{F}_{p}$ be the usual trace map and fix $\theta=\exp (2 \pi i / p)$, a primitive $p$ th root of unity. Put

$$
\delta=\left(e, \frac{q-1}{p-1}\right) \quad \text { and } \quad R=\frac{1}{\delta} \cdot \frac{q-1}{p-1},
$$

and let $C_{e}$ denote the group of $e$ th powers in $\mathbb{F}_{q}^{*}$. The Gauss periods are

$$
\eta_{j}=\sum_{x \in C_{e}} \theta^{\operatorname{Tr} g^{j} x} \quad(1 \leq j \leq e)
$$

and satisfy the period polynomial

$$
\Phi(x)=\prod_{j=1}^{e}\left(x-\eta_{j}\right) .
$$

In the classical case $q=p$, Gauss showed that $\Phi(x)$ is irreducible over $\mathbb{Q}$ and determined its coefficients for small values of $e$ and $f$. In 1982 I determined how to compute the beginning coefficients of $\Phi(x)$ for the classical case when $f$ is fixed [4]. (See also [3].)

G. Myerson [7] has shown that for the general case $q \neq p, \Phi(x)$ splits over $\mathbb{Q}$ into $\delta$ factors, each of degree $e / \delta$. To be precise,

$$
\Phi(x)=\prod_{w=1}^{\delta} \Phi^{(w)}(x),
$$

where

$$
\Phi^{(w)}(x)=\prod_{k=0}^{e / \delta-1}\left(x-\eta_{w+k \delta}\right) \quad(1 \leq w \leq \delta) .
$$


Each of the factors $\Phi^{(w)}(x)$ is irreducible or a power of an irreducible polynomial over $\mathbb{Q}$. Explaining patterns of additional reducibility that occur for $\Phi^{(w)}(x)$ was the primary focus of recent work of mine [5]. Here I consider instead the problem of computing the coefficients of a given factor $\Phi^{(w)}(x)$, particularly when $w=\delta$. I determine in Section 3 how to compute the beginning coefficients of the last factor $\Phi^{(\delta)}(x)$ in (3) in a manner analogous to that known for the case $q=p$ in [3] and [4].

2. Computations of the coefficients of $\Phi^{(w)}(x)$. Here I first express the coefficients $a_{r}=a_{r}(w)$ of a factor

$$
\Phi^{(w)}(x)=x^{e / \delta}+a_{1} x^{e / \delta-1}+\ldots+a_{e / \delta}
$$

of the period polynomial (3) for fixed $w, 1 \leq w \leq \delta$, in terms of the symmetric power sums

$$
S_{n}=S_{n}(w)=\sum_{k=0}^{e / \delta-1}\left(\eta_{k \delta+w}\right)^{n} .
$$

Specifically, this is given by Newton's identities

$$
S_{r}+a_{1} S_{r-1}+a_{2} S_{r-2}+\ldots+a_{r-1} S_{1}+r a_{r}=0 \quad(1 \leq r \leq e / \delta) .
$$

To obtain a computationally practical formula for $S_{n}$, I introduce a certain counting function $t_{w}(n)$ as follows. For a fixed integer $w$ and any $n>0$, let $t_{w}(n)$ count the number of $n$-tuples $\left(x_{1}, \ldots, x_{n}\right)$ in $\left(C_{e}\right)^{n}$ for which $\operatorname{Tr}\left(g^{w}\left(x_{1}+\ldots+x_{n}\right)\right)=0$. I assert that

$$
S_{n}(w)=-R f^{n-1}+p(e / \delta) t_{w}(n) /(p-1)
$$

in (6) for $n>0$. To see this, first write $\delta=c(q-1) /(p-1)+$ he for integers $h$ and $c$. Then for any fixed $j, g^{\delta j+w}=G^{c j} g^{h e j+w}, 0 \leq j<e / \delta$, where $G=g^{(q-1) /(p-1)}$ generates $\mathbb{F}_{p}^{*}$. Now $t_{w}(n)$ also counts the number of $n$-tuples in $\left(C_{e}\right)^{n}$ with $\operatorname{Tr}\left(g^{\delta j+w}\left(x_{1}+\ldots+x_{n}\right)\right)=0$ since $\operatorname{Tr}\left(g^{\delta j+w}\left(x_{1}+\ldots+x_{n}\right)\right)=$ $G^{c j} \operatorname{Tr}\left(g^{w} g^{h e j}\left(x_{1}+\ldots+x_{n}\right)\right)$, so

$$
t_{v}(n)=t_{w}(n) \quad \text { for } v \equiv w(\bmod \delta) .
$$

In particular, $t_{w}(n)$ counts the number of ones $\left(\theta^{0}\right)$ occurring in the multinomial expansion of any $\eta_{k \delta+w}^{n}=\left(\sum_{x \in C_{e}} \theta^{\operatorname{Tr} g^{k \delta+w} x}\right)^{n}$. A simple counting argument similar to that used in [4, p. 349] now yields (8). In particular, one finds $a_{1}=R-p(e / \delta) t_{w}(1) /(p-1)$ from (7). A much tidier expression for $a_{1}$ is given below.

Proposition 1. For $1 \leq w \leq \delta$, let $T(w)$ count the number of times $\operatorname{Tr} g^{\delta \nu+w}=0$ for $1 \leq \nu \leq R$. Then $a_{1}=R-p T(w)$ in $(5)$.

Proof. It suffices to show that $t_{w}(1)=\delta(p-1) T(w) / e$. I first assert that $T(w)$ also counts the number of times $\operatorname{Tr} g^{l \delta \nu+w}=0(1 \leq \nu \leq R)$ 
for any integer $l$ prime to $R$. To see this, note that for $\nu \equiv \nu^{\prime}(\bmod R)$, $\operatorname{Tr} g^{\delta \nu+w}=0 \Leftrightarrow \operatorname{Tr} g^{\delta \nu^{\prime}+w}=0$, as $g^{\delta \nu^{\prime}+w}=g^{\delta \nu+w} \cdot G^{t}$ if $\nu^{\prime}=\nu+t R$. Since $l \nu$ runs through a complete set of residues modulo $R$ for $1 \leq \nu \leq R$, the assertion about $T(w)$ follows. In particular, $T(w)$ counts the number of times $\operatorname{Tr} g^{e \nu+w}=0(1 \leq \nu \leq R)$ since $(e / \delta, R)=1$. Hence $\delta(p-1) T(w) / e$ counts the number of times $\operatorname{Tr} g^{e \nu+w}=0(1 \leq \nu \leq(q-1) / e)$ which is just $t_{w}(1)$.

An immediate consequence of Proposition 1 is the following reducibility criterion for $\Phi^{(w)}(x)$.

Corollary 1. If $T(w)=0$ then $\Phi^{(w)}(x)$ is irreducible over $\mathbb{Q}$.

Proof. When $T(w)=0, a_{1}=R$ is prime to $e / \delta$, the degree of $\Phi^{(w)}(x)$. Hence, since $\Phi^{(w)}(x)$ is some power of an irreducible, $\Phi^{(w)}(x)$ itself must be irreducible. (This is essentially how Myerson argues the irreducibility of $\Phi(x)$ when $\delta=1$ in $[7$, Theorem 6].)

A few comments are in order when $p \equiv 1(\bmod f)$. Then $e$ is a multiple of $(q-1) /(p-1)$ so $\delta=(q-1) /(p-1), R=1$ and $e / \delta=(p-1) / f$. In particular $C_{e} \subseteq \mathbb{F}_{p}^{*}$, so $t_{w}(n)$ counts the number of tuples $\left(x_{1}, \ldots, x_{n}\right)$ in $C_{e}^{n}$ satisfying $\operatorname{Tr} g^{w}\left(x_{1}+\ldots+x_{n}\right)=\left(\operatorname{Tr} g^{w}\right)\left(x_{1}+\ldots+x_{n}\right)=0$. If $\operatorname{Tr} g^{w} \neq 0$ then $t_{w}(n)$ coincides with the counting function $\beta_{p, f}(n)$ in [3, p. 392], so $S_{n}(w)=\left(-f^{n}+p \beta_{p, f}(n)\right) / f$ in $(8)$, and hence $\Phi^{(w)}(x)$ is the ordinary cyclotomic period polynomial for $\mathbb{F}_{p}$ of degree $e / \delta[4$, p. 349]. On the other hand, if $\operatorname{Tr} g^{w}=0$ then $t_{w}(n)=f^{n}$ so $S_{n}(w)=(e / \delta) f^{n}$ in (8), and thus $\Phi^{(w)}(x)=(x-f)^{e / \delta}$. To summarize, I have shown:

Proposition 2. Suppose $p \equiv 1(\bmod f)$ and $1 \leq w \leq \delta$. If $\operatorname{Tr} g^{w}=0$ then $\Phi^{(w)}(x)=(x-f)^{e / \delta}$ else $\Phi^{(w)}(x)$ is the ordinary cyclotomic period polynomial of degree $e / \delta$.

In the general case $p \not \equiv 1(\bmod f)$ there seems to be no nice interpretation of $t_{w}(n)$ as above, except for special values of the form $w=k \delta / m$ for fixed $m \mid \delta$ and $1 \leq k \leq m$. In the next section, I treat the simplest such case $w=\delta$ and describe how to compute the beginning coefficients of $\Phi^{(\delta)}(x)$ in a manner analogous to that for ordinary cyclotomic period polynomials $[3,4]$. The methods used may be extended to handle other cases $w=k \delta / m$, with $m>1$, but not without additional difficulties.

3. Beginning coefficients of the last factor $\Phi^{(\delta)}(x)$. Retaining the notation of the previous section, I determine here how to compute the beginning coefficients of the last factor $\Phi^{(\delta)}(x)$ in (5), or equivalently those of

$$
\mathbf{F}(X)=X^{e / \delta} \Phi^{(\delta)}\left(X^{-1}\right)=1+a_{1} X+\ldots+a_{e / \delta} X^{e / \delta},
$$


for fixed $f>1$. My goal is to generalize the results known in the classical case $q=p[3,4]$ by exhibiting a suitable counting function which coincides with $t_{\delta}(n)$ in (8) for all sufficiently large $p$. For this purpose fix an integer $r$ prime to $f$ satisfying $1 \leq r \leq f$, say with $\operatorname{ord}_{f} r=b$, and consider primes $p \equiv r$ $(\bmod f)$. One finds then that $e / \delta=(p-1) /(p-1, f)$ and $R=f /(p-1, f)$. Further, all such primes have common decomposition field $K$ in $\mathbb{Q}(\zeta)$, where $\zeta=\exp (2 \pi i / f)$, with $[\mathbb{Q}(\zeta): K]=b$. (The field $K$ is that subfield of $\mathbb{Q}(\zeta)$ fixed by the action $\zeta \rightarrow \zeta^{r}$.) For $n>0$, let $\beta_{K}(n)$ count the number of times $\operatorname{Tr}_{\mathbb{Q}(\zeta) / K}\left(x_{1}+\ldots+x_{n}\right)=0$ for choice of $f$-roots of unity $x_{1}, \ldots, x_{n}$ lying in $\mathbb{Q}(\zeta)$. That $\beta_{K}(n)=t_{\delta}(n)$ for large enough $p$ is demonstrated next.

Proposition 3. If $p>(b n)^{\phi(f) / b}$ and $p \nmid a$, then $t_{\delta}(n)=\beta_{K}(n)$. (Here $\phi$ is Euler's totient function.)

Proof. Since $p^{b} \equiv 1(\bmod f)$ the element $g^{e}$ lies in $\mathbb{F}_{p^{b}} \subseteq \mathbb{F}_{q}$. Thus, one may identify $\mathbb{F}_{p^{b}} / \mathbb{F}_{p}$ as the residue field extension at $p$ for the extension $\mathbb{Q}(\zeta) / K$ for some prime $P$ lying above $p$ in $\mathbb{Q}(\zeta)$ where $g^{e}$ corresponds to $\zeta$ $(\bmod P)$. The condition $p>(b n)^{\phi(f) / b}$ ensures that for $0 \leq \alpha_{i}<f(1 \leq i \leq$ $n), \operatorname{Tr}_{\mathbb{F}_{p^{b}} / \mathbb{F}_{p}}\left(g^{e \alpha_{1}}+\ldots+g^{e \alpha_{n}}\right) \neq 0$ unless $\operatorname{Tr}_{\mathbb{Q}(\zeta) / K}\left(\zeta^{\alpha_{1}}+\ldots+\zeta^{\alpha_{n}}\right)=0$ otherwise $P \mid \operatorname{Tr}_{\mathbb{Q}(\zeta) / K}\left(\zeta^{\alpha_{1}}+\ldots+\zeta^{\alpha_{n}}\right)$, which implies

$$
p \leq\left|N_{K / \mathbb{Q}}\left(\operatorname{Tr}_{\mathbb{Q}(\zeta) / K}\left(\zeta^{\alpha_{1}}+\ldots+\zeta^{\alpha_{n}}\right)\right)\right| \leq(b n)^{\phi(f) / b} .
$$

Thus $\beta_{K}(n)$ counts the number of times $\operatorname{Tr}_{\mathbb{F}_{p^{b}} / \mathbb{F}_{p}}\left(x_{1}+\ldots+x_{n}\right)=0$ for $x_{i} \in C_{e}(1 \leq i \leq n)$. Now, in addition,

$$
\operatorname{Tr}_{\mathbb{F}_{q} / \mathbb{F}_{p}}\left(x_{1}+\ldots+x_{n}\right)=\frac{a}{b} \operatorname{Tr}_{\mathbb{F}_{p^{b}} / \mathbb{F}_{p}}\left(x_{1}+\ldots+x_{n}\right) \quad \text { for } x_{i} \in C_{e} .
$$

Hence, if $p \nmid a$ then $\beta_{K}(n)=t_{0}(n)$, which is the same as $t_{\delta}(n)$ by (9).

I should remark that the finite set $\xi_{n}$ of exceptional primes for which $t_{\delta}(n)>\beta_{K}(n)$ can be determined in a manner analogous to the case $q=p[3]$ by finding the rational primes dividing any of the norms $N_{K / \mathbb{Q}}\left(\operatorname{Tr}_{\mathbb{Q}(\zeta) / K}\left(\zeta^{\alpha_{1}}\right.\right.$ $\left.+\ldots+\zeta^{\alpha_{n}}\right)$ ), where $0 \leq \alpha_{i}<f, 1 \leq i \leq n$.

In general the counting function $\beta_{K}(n)$ is difficult to determine. A simple closed formula for $\beta_{K}(n)$ in certain special cases is given by the following two propositions.

Proposition 4. If $f=l$, a prime, then

$$
\beta_{K}(n)= \begin{cases}b^{n(l-1) / l} \frac{n !}{(n / l) ![(b n / l) !]^{(l-1) / b}} & \text { if } l \mid n, \\ 0 & \text { otherwise. }\end{cases}
$$

Proof. When $l=2$, one finds $b=1, K=\mathbb{Q}$ and $\zeta=-1$. An easy counting argument shows $\beta_{\mathbb{Q}}(n)=0$ or $\left(\begin{array}{c}n \\ n / 2\end{array}\right)$ according as $n$ is odd or even. Now consider the case $l$ is an odd prime, and observe that then an integral linear combination $c_{0}+c_{1} \zeta+\ldots+c_{l-1} \zeta^{l-1}$ equals zero if and only if 
$c_{0}=c_{1}=\ldots=c_{l-1}$. A straightforward argument shows that $\operatorname{Tr}_{\mathbb{Q}(\zeta) / K}\left(\zeta^{\alpha_{1}}+\right.$ $\left.\ldots+\zeta^{\alpha_{n}}\right)=0$ for $0 \leq \alpha_{i}<l(1 \leq i \leq n)$ if and only if $l \mid n$ and $n / l$ of the $\alpha$ 's are zero, with the remaining $(n / l)(l-1) \alpha$ 's equally distributed among the $(l-1) / b$ cosets of the multiplicative subgroup $\langle r\rangle$ in $\mathbb{Z}_{l}^{*}$. For a fixed choice of coset representatives $T=\left\{t_{1}, \ldots, t_{(l-1) / b}\right\}$ there are

$$
M=\frac{n !}{(n / l) ![(b n / l) !]^{(l-1) / b}}
$$

ways to choose the $(n / l)(l-1)$ non-zero $\alpha$ 's from among $T$ so that $\operatorname{Tr}_{\mathbb{Q}(\zeta) / K}\left(\zeta^{\alpha_{1}}+\ldots+\zeta^{\alpha_{n}}\right)=0$. As each coset is of size $b=\operatorname{ord}_{l} r$ and the choice of a given $\alpha_{i} \neq 0$ in $\operatorname{Tr}_{\mathbb{Q}(\zeta) / K}\left(\zeta^{\alpha_{1}}+\ldots+\zeta^{\alpha_{n}}\right)$ depends only on the coset it represents, one finds that $\beta_{K}(n)=b^{n(l-1) / l} M$ when $l \mid n$. The result stated in the proposition now follows.

Proposition 5. (i) For $f=4$ and $r=1$,

$$
\beta_{K}(n)= \begin{cases}\frac{(n !)^{2}}{[(n / 2) !]^{4}} & \text { if } 2 \mid n, \\ 0 & \text { if } 2 \nmid n .\end{cases}
$$

(ii) For $f=4$ and $r=3, \beta_{K}(n)=\left(\begin{array}{c}2 n \\ n\end{array}\right)$.

Proof. In view of the result of Proposition 2, the counting function $\beta_{K}(n)$ in statement (i) is what Gupta and Zagier call $\beta_{4}(n)$ in [3]. Thus statement (i) is just equation (5) in [3, Theorem 2], which was first observed by D. H. and E. Lehmer [6].

To verify statement (ii) of the proposition note that $K=\mathbb{Q}$ here, so $\operatorname{Tr}_{\mathbb{Q}(\zeta) / K}\left(\zeta^{\alpha}\right)=0$ if $\alpha$ is odd, else equals 2 or -2 according as $4 \mid \alpha$ or $2 \| \alpha$. Begin by encoding each fourth root of unity by a pair of ones and minus ones, so that $\zeta$ corresponds to the pair $(1,-1), \zeta^{2}$ to $(-1,-1), \zeta^{3}$ to $(-1,1)$ and $\zeta^{4}$ to $(1,1)$. The encoding is such that the trace $\operatorname{Tr}_{\mathbb{Q}(\zeta) / K}\left(\zeta^{\alpha}\right)$ equals the sum of its corresponding pair of values. Moreover, one may identify an $n$-tuple $\left(x_{1}, \ldots, x_{n}\right)$ of fourth roots of unity by a unique $2 n$-tuple $\left(y_{1}, y_{2}, \ldots, y_{2 n-1}, y_{2 n}\right)$ consisting of ones and minus ones, where $x_{j}$ corresponds to the pair $\left(y_{2 j-1}, y_{2 j}\right)(1 \leq j \leq n)$ as described, and vice versa. The correspondence is such that each tuple $\left(x_{1}, \ldots, x_{n}\right)$ with $\operatorname{Tr}_{\mathbb{Q}(\zeta) / K}\left(x_{1}+\right.$ $\left.\ldots+x_{n}\right)=0$ yields a tuple $\left(y_{1}, \ldots, y_{2 n}\right)$ with $y_{1}+\ldots+y_{2 n}=0$, and vice versa. Thus $\beta_{K}(n)=\left(\begin{array}{c}2 n \\ n\end{array}\right)$, the number of ways to fill a $2 n$-tuple with an equal number of ones and minus ones.

Thus statement (ii) is verified and the proof of the proposition is now complete.

Now let $h$ be the smallest positive integer for which $\beta_{K}(h) \neq 0$. Using (7), (8) and Proposition 2, one may obtain the following generalization of Theorem 1 in [4]. Since the argument is identical, I shall omit it here. 
TheOREM 1. For all sufficiently large primes $p \equiv r(\bmod f)$, the coefficient $a_{s}$ of the polynomial $\Phi^{(\delta)}(x)$ in (5) (or $\mathbf{F}(X)$ in (10)) satisfies $a_{s}=\mho_{s}(p)$, where $\mho_{s}$ is a polynomial of degree $[s / h]$ in $p$.

The next examples illustrate the result above.

EXAmple 1. Consider the case $f=3$ and $r=2$ with $q=p^{2}$ above in Theorem 1 , so $R=3$ and $e / \delta=p-1$ in (8). The decomposition field $K=\mathbb{Q}$ with

$$
\beta_{K}(n)= \begin{cases}4^{n / 3}\left(\begin{array}{c}
n \\
n / 3
\end{array}\right) & \text { if } 3 \mid n, \\
0 & \text { otherwise }\end{cases}
$$

from Proposition 4 , so $h=3$. One finds the following expressions for the coefficients $a_{s}(1 \leq s \leq 8)$ for $\Phi^{(\delta)}(x)$ from (7) and (8):

$$
\begin{array}{lc}
a_{1}=3, \quad a_{2}=9, \quad a_{3}=-(4 p-27) & \text { for } p>2, \\
a_{4}=-(12 p-81), \quad a_{5}=-(36 p-243), \quad a_{6}=8 p^{2}-148 p+ & 729 \\
a_{7}=24 p^{2}-444 p+2187, \quad a_{8}=72 p^{2}-1332 p+6561 & \text { for } p>5,
\end{array}
$$

One observes that $\Phi^{(\delta)}(x)$ is always irreducible from Corollary 1.

EXAmple 2. Consider next the case $f=8$ and $r=3$ or 7 with $q=p^{2}$ in Theorem 1 , so $R=4$ and $e / \delta=(p-1) / 2$ in (8). The decomposition field $K$ is $\mathbb{Q}(\sqrt{-2})$ or $\mathbb{Q}(\sqrt{2})$, respectively, but it is easy to verify that the counting function $\beta_{K}(n)$ is the same in each case. For the first few values, one computes $\beta_{K}(1)=2, \beta_{K}(2)=14, \beta_{K}(3)=68$ and $\beta_{K}(4)=454$. Thus $h=1$ and one finds the following expression for the coefficients $a_{s}(1 \leq s \leq 4)$ for $\Phi^{(\delta)}(x)$ from (7) and (8).

$$
\begin{array}{ll}
a_{1}=-(p-4), \quad a_{2}=\frac{1}{2}\left(p^{2}-15 p+48\right) & \text { for } p>3, \\
a_{3}=-\frac{1}{6}\left(p^{3}-33 p^{2}+296 p-960\right) & \text { for } p>7 \text { and } \\
a_{4}=\frac{1}{24}\left(p^{4}-58 p^{3}+1043 p^{2}-8306 p+26880\right) & \text { for } p>19 .
\end{array}
$$

The pattern of these coefficients is exhibited below for primes $p<23$.

\begin{tabular}{rl}
\hline$p$ & Factor $\Phi^{(\delta)}(x)$ \\
\hline 3 & $x+1$ \\
7 & $x^{3}-3 x^{2}-4 x+13$ \\
11 & $x^{5}-7 x^{4}+2 x^{3}+61 x^{2}-123 x+67$ \\
19 & $x^{9}-15 x^{8}+62 x^{7}+65 x^{6}-951 x^{5}+1585 x^{4}$ \\
& $\quad+616 x^{3}-1846 x^{2}-583 x-37$ \\
\hline
\end{tabular}


It is interesting to note that when $h>1$, the polynomial $\Phi^{(\delta)}(x)$ is irreducible for sufficiently large $p$ by Proposition 3 and the corollary to Proposition 1. In particular, $h>1$ whenever $f$ is square-free, since then $\operatorname{Tr}_{\mathbb{Q}(\zeta) / K}\left(\zeta^{\alpha}\right) \neq 0$ for any integer $\alpha$.

To generalize Theorem 1 of S. Gupta and D. Zagier [3], I next introduce the rational power series

$$
B_{K}(X)=\exp \left(-R \sum_{n=1}^{\infty} \beta_{K}(n) \frac{X^{n}}{n}\right)
$$

and

$$
A_{K, r}(X)=\exp \left(\frac{r}{f} \log B_{K}(X)-\frac{R}{f} \log (1-f X)\right),
$$

defined in terms of the counting function $\beta_{K}(n)$.

The argument in the proof of Theorem 1 of [3] extends in a straightforward manner to yield the following general result here.

Theorem 2. The power sums $B_{K}(X)$ and $A_{K, r}(X)$ above lie in $\mathbb{Z}[[X]]$ and satisfy

$$
(1-f X)^{R} A_{K, r}(X)^{f}=B_{K}(X)^{r} .
$$

For any $N>0$ there is a constant $p_{0}(N)$ such that for all primes $p \equiv r$ $(\bmod f)$ with $p>p_{0}(N)$,

$$
\mathbf{F}(X) \equiv A_{K, r}(X) B_{K}(X)^{(p-r) / f}\left(\bmod X^{N}\right) .
$$

For Example 1, the relevant power series (11) and (12) are given by

$$
B_{K}(X)=1-12 X^{3}-48 X^{6}+\ldots
$$

and

$$
A_{K, 2}(X)=1+3 X+9 X^{2}+19 X^{3}+57 X^{4}+171 X^{5}+\ldots
$$

respectively.

In Example 2, the power series (11) is given by

$$
B_{K}(X)=1-8 X+4 X^{2}+48 X^{3}-62 X^{4}+\ldots ;
$$

the corresponding series (12) are

$$
A_{K, 3}(X)=1+X+6 X^{2}+57 X^{3}+411 X^{4}+\ldots
$$

and

$$
A_{K, 7}(X)=1-3 X-4 X^{2}+27 X^{3}+98 X^{4}+\ldots
$$

For the case $f=4$ and $r=3$, one has $R=2, K=\mathbb{Q}$ and $e / \delta=(p-1) / 2$. From Proposition 3 , for primes $p \equiv 3(\bmod 4)$ and not dividing $a$, one finds $t_{\delta}(n)=\beta_{K}(n)$ for $1 \leq n \leq(p-1) / 2$. In such cases one may take $N=(p+1) / 2$ in (13) which completely determines $\mathbf{F}(X)$ or $\Phi^{(\delta)}(x)$. It is even possible to find a closed form formula for the coefficients $a_{s}$ in (10); 
namely, $a_{s}=(-1)^{s}\left(\begin{array}{c}p-1-s \\ s\end{array}\right)$ for $1 \leq s \leq(p-1) / 2$. This result is proved in the section to follow. (Incidentally, if $p \mid a$ here, then it is easy to show that $\Phi^{(\delta)}(x)=(x-4)^{(p-1) / 2}$ since $t_{\delta}(n)=4^{n}$.)

4. The case $f=4$ and $r=3$. In order to derive the closed form formula mentioned at the end of the last section, the following well-known result will be needed.

Lemma. Let $d$ be a positive integer. For any polynomial $q(x)$ of degree less than $d$,

$$
\sum_{n=0}^{d}(-1)^{n}\left(\begin{array}{l}
d \\
n
\end{array}\right) q(n)=0 .
$$

Returning to the situation at hand, first observe that the power series

$$
C(X)=\exp \left(-\frac{1}{2} \sum_{n=1}^{\infty}\left(\begin{array}{c}
2 n \\
n
\end{array}\right) \frac{X^{n}}{n}\right)
$$

satisfies

$$
\frac{C^{\prime}(X)}{C(X)}=-\frac{1}{2} \sum_{n=1}^{\infty}\left(\begin{array}{c}
2 n \\
n
\end{array}\right) X^{n-1}=\frac{1}{2 X}\left(1-\frac{1}{\sqrt{1-4 X}}\right) .
$$

One finds then $C(X)=\frac{1}{2}(1+\sqrt{1-4 X})$. In particular, from Proposition 5(ii), the power series

$$
B_{K}(X)=C(X)^{4}=\frac{1}{2}\left(1-4 X+2 X^{2}+(1-2 X) \sqrt{1-4 X}\right)
$$

and

$$
A_{K, 3}(X)=\frac{C^{3}(X)}{\sqrt{1-4 X}}=\frac{1}{2}\left(1-X+\frac{1-3 X}{\sqrt{1-4 X}}\right)
$$

in (11) and (12), so

$$
\mathbf{F}(X) \equiv A_{K, 3}(X) B_{K}(X)^{(p-3) / 4} \equiv \frac{C(X)^{p}}{\sqrt{1-4 X}}\left(\bmod X^{(p+1) / 2}\right)
$$

in (13) where $p \nmid a$. But

$$
\begin{aligned}
(1-4 X)^{-1 / 2} C(X)^{p} & \\
& =2^{-p}(1-4 X)^{-1 / 2} \sum_{n=0}^{p}\left(\begin{array}{l}
p \\
n
\end{array}\right)(1-4 X)^{n / 2} \\
& =2^{-p} \sum_{n=0}^{p}\left(\begin{array}{l}
p \\
n
\end{array}\right)(1-4 X)^{(n-1) / 2} \\
& =2^{-p} \sum_{s=0}^{\infty}(4 X)^{s} \sum_{n=0}^{p} \frac{(-1)^{s}}{s !}\left(\begin{array}{l}
p \\
n
\end{array}\right)\left(\frac{n-1}{2}\right)\left(\frac{n-3}{2}\right) \ldots\left(\frac{n-2 s+1}{2}\right),
\end{aligned}
$$


so the congruence above yields

$$
a_{s}=\frac{(-1)^{s}}{2^{p-2 s} s !} \sum_{n=0}^{p}\left(\begin{array}{l}
p \\
n
\end{array}\right)\left(\frac{n-1}{2}\right)\left(\frac{n-3}{2}\right) \ldots\left(\frac{n-2 s+1}{2}\right)
$$

in (10) for $1 \leq s \leq(p-1) / 2$. Now Moriarty's identity (2.73) in [2] implies that

$$
\frac{1}{2^{p-2 s}} \sum_{\substack{n=0 \\
n \text { odd }}}^{p}\left(\begin{array}{l}
p \\
n
\end{array}\right)\left(\begin{array}{c}
\frac{n-1}{2} \\
s
\end{array}\right)=\frac{1}{2}\left(\begin{array}{c}
p-s-1 \\
s
\end{array}\right) .
$$

Since

$$
\sum_{\substack{n=0 \\
n \text { odd }}}^{p}\left(\begin{array}{l}
p \\
n
\end{array}\right)\left(\begin{array}{c}
\frac{n-1}{2} \\
s
\end{array}\right)=\sum_{\substack{n=0 \\
n \text { even }}}^{p}\left(\begin{array}{c}
p \\
n
\end{array}\right)\left(\begin{array}{c}
\frac{n-1}{2} \\
s
\end{array}\right) \quad \text { for } s<p
$$

by the Lemma, it follows from (14) that the coefficients $a_{s}$ in (10) actually satisfy

$$
a_{s}=(-1)^{s}\left(\begin{array}{c}
p-s-1 \\
s
\end{array}\right) \quad(1 \leq s \leq(p-1) / 2)
$$

when $p \nmid a$. In view of the parenthetical remark made at the end of Section 3, I have shown

Proposition 6. Let $f=4$ and $p \equiv 3(\bmod 4)$ be prime. If $p \nmid a$ then

$$
\Phi^{(\delta)}(x)=\sum_{s=0}^{(p-1) / 2}(-1)^{s}\left(\begin{array}{c}
p-s-1 \\
s
\end{array}\right) x^{(p-1) / 2-s}
$$

else

$$
\Phi^{(\delta)}(x)=(x-4)^{(p-1) / 2} .
$$

This concludes the discussion of the special case $f=4$ and $r=3$.

\section{References}

[1] Z. Borevich and I. Shafarevich, Number Theory, Academic Press, New York, 1966.

[2] G. P. Egorychev, Integral Representations and the Computation of Combinatorical Sums, Transl. Math. Monographs 39, Amer. Math. Soc., Providence, 1984.

[3] S. Gupta and D. Zagier, On the coefficients of the minimal polynomial of Gaussian periods, Math. Comp. 60 (1993), 385-398.

[4] S. Gurak, Minimal polynomials for Gauss circulants, Pacific J. Math. 102 (1982), 347-353.

[5] - Factors of period polynomials for finite fields, I, to appear.

[6] D. H. Lehmer and E. Lehmer, Cyclotomy with short periods, Math. Comp. 41 (1983), 743-758. 
[7] G. Myerson, Period polynomials and Gauss sums for finite fields, Acta Arith. 39 (1981), 251-264.

DEPARTMENT OF MATHEMATICS AND COMPUTER SCIENCE UNIVERSITY OF SAN DIEGO

5998 ALCALÁ PARK

SAN DIEGO, CALIFORNIA 92110-2492

U.S.A.

Received on 25.4.1994

and in revised form on 14.2.1995 